\title{
A FILOSOFIA ASSISTENCIAL DA MATERNIDADE DE UM HOSPITAL UNIVERSITÁRIO NA VISÃO DOS ACADÊMICOS
}

\author{
Marisa Monticelli, Odaléa Maria Brüggemann², Isabelle Christine Guerini ${ }^{3}$ Antonio Fernando Boing ${ }^{4}$, \\ Maria de Fátima Padilha ${ }^{5}$ Viviane Bergler Fernandes ${ }^{6}$
}

${ }^{1}$ Doutora em Enfermagem. Docente do Departamento de Enfermagem e do Programa de Pós-Graduação em Enfermagem (PEN) da Universidade Federal de Santa Catarina (UFSC). Santa Catarina, Brasil. E-mail: marisa@ccs.ufsc.br

${ }^{2}$ Doutora em Tocoginecologia. Docente do Departamento de Enfermagem e do PEN/UFSC. Santa Catarina, Brasil. E-mail: odalea@ccs.ufsc.br

${ }^{3}$ Acadêmica de Graduação em Enfermagem da UFSC. Bolsista PIBIC/CNPq. Santa Catarina, Brasil. E-mail: iguerini@yahoo. com.br

${ }^{4}$ Doutor em Ciências Odontológicas. Docente do Departamento de Saúde Pública da UFSC. Santa Catarina, Brasil. E-mail: boing@ccs.ufsc.br

${ }^{5}$ Enfermeira do Hospital Universitário (HU) da UFSC. Santa Catarina, Brasil. E-mail: fatimahu2003@yahoo.com.br

${ }^{6}$ Assistente Social do HU/UFSC. Santa Catarina, Brasil. E-mail: vivibergler@yahoo.com.br

RESUMO: Estudo transversal exploratório, que teve por objetivo avaliar a filosofia assistencial da maternidade de um hospital universitário, sob a ótica dos acadêmicos. A amostra foi composta por 47 alunos de enfermagem e 47 alunos de medicina. Os dados foram coletados em 2008, por meio de questionário. A análise englobou estatística descritiva e teste qui-quadrado. Os resultados evidenciaram que os princípios relacionados com a filosofia geral da assistência e com os direitos dos usuários são implementados em quase todos os setores, enquanto os relacionados com a integração entre os profissionais e entre os setores são os que apresentam maiores fragilidades. De forma geral os acadêmicos conhecem a filosofia e consideram que a mesma é implementada na maioria dos setores, com destaque para o centro obstétrico, o alojamento conjunto e a central de incentivo ao aleitamento materno. Contudo, também ressaltam que a operacionalização da filosofia, em sua totalidade, ainda permanece um desafio a ser superado.

DESCRITORES: Parto humanizado. Serviços de saúde materna. Avaliação de serviços de saúde. Estudantes de medicina. Estudantes de enfermagem.

\section{MATERNITY CARE PHILOSOPHY IN A UNIVERSITY HOSPITAL: THE ACADEMIC VIEW}

\begin{abstract}
The objective of this cross-sectional exploratory study was to evaluate maternity care philosophy in a university hospital, from the academic perspective. The sample was composed of 47 nursing students and 47 medical students. The data was collected in 2008 via questionnaire. Data analysis encompassed descriptive statistics and the chi-squared test. The results evidenced that the principles related to the general care philosophy and the rights of users are implemented in almost every sector of the university hospital, while those related to integration among professionals and among sectors present the greatest fragilities. In general, health care students understand such philosophy and consider it to be implemented in every sector of the hospital, especially the obstetrics center, shared in-patient ward, and the center for incentivizing maternal breast-feeding. However, we also point out that making philosophy operational in its totality still remains a challenge to be faced and achieved.

DESCRIPTORS: Humanizing delivery. Maternal health services. Health services evaluation. Students, medical. Students, nursing.

\section{LA FILOSOFÍA ASISTENCIAL DE LA MATERNIDAD DE UN HOSPITAL UNIVERSITARIO DESDE LA VISIÓN DE LOS ESTUDIANTES}

\begin{abstract}
RESUMEN: Es un estudio transversal exploratorio, cuyo objetivo fue evaluar la filosofía asistencial de la maternidad de un hospital universitario, desde la visión de los estudiantes. La muestra se formó con 47 alumnos de enfermería y 47 alumnos de medicina. Los datos fueron recolectados en 2008 por medio de un cuestionario. El análisis consideró la estadística descriptiva y el teste chi-cuadrado. Los resultados mostraron que los principios relacionados con la filosofía general de la asistencia y con los derechos de los usuarios son implementados en casi todos los sectores, mientras que los que se relacionan con la integración entre los profesionales y entre los sectores son los que presentan mayores fragilidades. Los estudiantes, generalmente, conocen la filosofía asistencial y consideran que ella es implementada en la mayoría de los sectores, con destaque especial para el centro obstétrico, el alojamiento colectivo y la central de estímulo al amamantamiento materno. Sin embargo, también resaltan que la operatividad de la filosofía aún es un desafío a ser superado.

DESCRIPTORES: Parto humanizado. Servicios de salud materna. Evaluación de servicios de salud. Estudiantes de medicina. Estudiantes de enfermería.
\end{abstract}




\section{INTRODUÇÃO}

Filosofias de atenção à saúde, bem como políticas de saúde, de modo geral, somente são materializadas quando ações dos diversos atores sociais são postas em evidência no cotidiano dos serviços e das organizações. ${ }^{1}$ Este pressuposto se encontra na base da preocupação epistemológica que dá sustentação à presente proposta investigativa.

A problematização da realidade que a orienta tem origem na própria dinâmica de trabalho de uma equipe multidisciplinar, especialmente instalada pela direção do Hospital Universitário (HU) da Universidade Federal de Santa Catarina (UFSC), em 1980, com a finalidade de definir os rumos conceituais, filosóficos e pedagógicos que sustentariam a maternidade da instituição - um setor que, à época, encontrava-se entre as prioridades institucionais e iniciava sua edificação física. Esta equipe trabalhou na proposta entre meados de 1980 a meados de 1990, tendo uma mesma composição representativa de profissionais da assistência e docência, contudo, alguns membros foram sendo substituídos, uma vez que o tempo decorrido de trabalho foi de uma década.

A equipe definiu algumas linhas mestras que pudessem amparar a idéia de inclusão e humanização da assistência ao parto e nascimento e que beneficiasse os clientes/usuários do serviço, em regime de cooperação interdisciplinar. Permanecia em sua base o ideário em torno de uma filosofia de atenção que respeitasse as práticas obstétricas recomendadas pela Organização Mundial da Saú$\mathrm{de}^{2} \mathrm{e}$ os fundamentos das evidências científicas na área da saúde. ${ }^{3} \mathrm{O}$ trabalho coletivo e interdisciplinar desta equipe culminou com a eleição de um conjunto de princípios filosóficos que passou a ser amplamente reconhecido como "a filosofia da maternidade", sendo colocada em prática desde o primeiro dia de abertura da maternidade, em 24 de outubro de 1995, permanecendo até os dias atuais. Esta filosofia possui 12 princípios, sendo que quatro deles dizem respeito a aspectos gerais da assistência, cinco estão relacionados com a integração entre os profissionais e os serviços, e três contemplam direitos dos usuários. Cabe destacar que todos esses conjuntos de princípios estão calcados em fundamentos da interdisciplinaridade e da humanização da assistência.

Para garantir a operacionalização dessa filosofia, o Grupo Interdisciplinar de Assessoria à Maternidade (GIAM), diretamente ligado à Direção Geral da instituição, vem desenvolvendo atividades e reflexões continuadas, visando assegurar esses princípios, seja no auxílio à formulação das rotinas dos setores que integram a maternidade, seja no acompanhamento de educação continuada, envolvendo os recursos humanos que assistem, ensinam ou têm formação acadêmica na maternidade do HU.

Contudo, todos os segmentos componentes do GIAM, ou seja, os representantes dos profissionais que atuam na assistência, os representantes dos docentes dos Departamentos de Ensino da UFSC que atuam diretamente na maternidade, e também as chefias de seções e serviços, além daqueles profissionais que estão diretamente envolvidos com a atenção aos usuários da maternidade, têm exteriorizado, cada vez com mais frequência, a necessidade de proceder a um processo avaliativo da aplicação dos princípios filosóficos à prática cotidiana dos serviços, que seja sistemático e com critérios amplos de validação. Afinal, todos esses participantes continuam acreditando que uma filosofia assistencial somente se materializa e " faz sentido" quando a mesma se concretiza em atos e acontecimentos cotidianos que reflitam a intencionalidade subjacente a tais práticas.

Avaliações nesta perspectiva vêm sendo realizadas durante todos estes anos de vigência da "filosofia da maternidade", mas na grande maioria, trata-se de levantamentos mais setorizados, esporádicos e com alcance de pequena escala, como por exemplo, os depoimentos dados por puérperas e acompanhantes (muitos deles, encaminhados espontaneamente e por escrito à Direção ou aos setores da maternidade, após a alta); o preenchimento de questionários de avaliação pelos acadêmicos da área da saúde; ou as narrativas obtidas de profissionais representantes das várias categorias profissionais que integram os serviços da maternidade, tanto em diálogos isolados, quanto em discussões temáticas em pequenos grupos.

Um exemplo mais completo sobre processos avaliativos dessa natureza e que foi conduzido de forma sistemática trata-se da pesquisa realizada por uma das enfermeiras que atuam no centro obstétrico da maternidade, e que resultou em uma dissertação de mestrado. ${ }^{4}$ Nesta pesquisa a autora buscou avaliar a incorporação da filosofia de atendimento da maternidade pelos profissionais do centro obstétrico. Dentre os principais achados a autora destacou que alguns profissionais realizam ações diárias de cumprimento aos princípios filosóficos que regem a maternidade e possuem condutas profissionais e interativas que se coadunam 
com os pressupostos humanísticos que embasam a filosofia. Entretanto, também apontou que algumas rotinas de atenção não são totalmente regidas por tal filosofia e também que vários profissionais da equipe obstétrica não reconhecem muitos dos princípios como balizadores da assistência, outros os desconhecem em sua inteireza e há ainda os profissionais que atuam conforme sua formação de origem, não reconhecendo a filosofia como um paradigma que norteia a vida da maternidade e a atenção obstétrica e neonatal.

É justamente pela escassez de resultados que permitam alinhavar um panorama mais concreto da aplicação de tais princípios, na prática da maternidade, que o presente estudo se justifica; especialmente no que se refere à percepção dos acadêmicos de enfermagem e de medicina, já que os mesmos desenvolvem atividades estudantis, de modo concomitante com as atividades assistenciais, nas dependências da maternidade. A visão dos demais sujeitos envolvidos, ou seja, os profissionais e os usuários da maternidade, é objeto de outras investigações paralelas que estão sendo realizadas pelos integrantes que compõem o GIAM.

Estudo anterior, desenvolvido em outro cenário assistencial, ${ }^{5}$ já concluiu, inclusive, que a percepção de estudantes de enfermagem, acerca de estratégias de atenção ao parto e nascimento, em nível hospitalar, é a de que a mulher está submetida a diferentes graus e tipos de violência, ainda que tais práticas sejam designadas como "de humanização", não condizendo, portanto, com as intenções filosóficas e políticas que, supostamente, dariam sustentação a tal proposta.

Assim, configura-se como objetivo desta pesquisa, avaliar a implementação da filosofia da maternidade do HU/UFSC sob a ótica dos acadêmicos de enfermagem e de medicina.

\section{METODOLOGIA}

Trata-se de uma pesquisa transversal exploratória, que foi desenvolvida na maternidade do HU/UFSC no ano de 2008.

A amostra foi intencional, composta por acadêmicos de enfermagem da $5^{\mathrm{a}}$ e $8^{\mathrm{a}}$ fases curriculares, e por acadêmicos da $11^{\text {a }}$ fase curricular do curso de medicina, que desenvolveram atividades teórico-práticas em um ou mais serviços da maternidade, no semestre 2008/1 e no semestre 2008/2. As atividades teórico-práticas desses alunos, ocorreram de modo mais concentrado nos setores de
Triagem Obstétrica (TO), Alojamento Conjunto (AC), Centro Obstétrico (CO), Central de Incentivo ao Aleitamento Materno (CIAM) e no Ambulatório de Tocoginecologia (AT), considerando-se as especificidades de cada curso.

De um total de 76 acadêmicos de enfermagem convidados e que poderiam compor a amostra (matriculados nos semestres 2008/1 e $2008 / 2$, especificamente na $5^{\mathrm{a}}$ e $8^{\mathrm{a}}$ fases) e de um total de 93 acadêmicos de medicina (matriculados nos mesmos semestres citados, porém na $11^{\text {a }}$ fase), participaram efetivamente da pesquisa 47 acadêmicos de enfermagem e 47 de medicina, totalizando então 94 acadêmicos.

Para a coleta de dados foi aplicado um questionário auto-respondido, que foi previamente testado com cinco acadêmicos de enfermagem e com cinco de medicina, e posteriormente reformulado. Tal questionário continha perguntas sobre a operacionalização dos 12 princípios filosóficos relacionados com os aspectos gerais da assistência (Princípios 1, 3, 10 e 12), relacionados com a integração entre os profissionais e os serviços (Princípios $6,7,8$, 9 e 11) e relacionados aos direitos dos usuários (Princípios 2, 4 e 5).

Os acadêmicos foram convidados a participar da pesquisa nas últimas duas semanas em que estiveram desenvolvendo atividades teórico-práticas na maternidade (nos dois semestres de 2008), para que tivessem subsídios para o preenchimento do questionário. Os que aceitaram participar entregaram o questionário preenchido diretamente para os pesquisadores responsáveis ou então na secretaria do Serviço de Tocoginecologia, localizado no $2^{\circ}$ andar do HU. Os questionários não possuíam dados de identificação, de modo que o anonimato dos participantes foi garantido durante todo o decorrer do processo investigativo.

Para o processo de organização e análise dos dados, todos os questionários foram codificados e digitados no programa Epi Info - versão 2002. Realizou-se análise estatística descritiva (distribuição absoluta e relativa) de todos os dados e o teste qui-quadrado para comparar as respostas dos acadêmicos de enfermagem e de medicina sobre a operacionalização de cada princípio filosófico, nos setores da maternidade. O nível de significância assumido foi de $5 \%$.

O projeto foi aprovado pelo Comitê de Ética em Pesquisa com Seres Humanos da UFSC, sob protocolo $\mathrm{N}^{\circ} 263 / 07$. Os aspectos que envolvem a pesquisa estão de acordo com a Resolução $\mathrm{N}^{\circ}$ 196/96 do Conselho Nacional de Saúde, sendo 
que os todos os acadêmicos de enfermagem e de medicina assinaram o Termo de Consentimento Livre e Esclarecido.

\section{RESULTADOS E DISCUSSÃO}

Os dados relativos às características sóciodemográficas dos acadêmicos indicam que a maioria dos acadêmicos de enfermagem era do sexo feminino $(95,7 \%)$ e os de medicina, do sexo masculino $(57,4 \%)$. Para ambas as áreas, predomina o intervalo de idade entre 20 e 24 anos $(74,5 \%$ enfermagem e $59,6 \%$ medicina). O estado marital também apresenta característica parecida, uma vez que, $83,0 \%$ dos acadêmicos de enfermagem e 95,7\% dos acadêmicos de medicina eram solteiros. Com relação ao período do curso frequentado, 80,9\% dos acadêmicos de enfermagem cursavam a $5^{\text {a }}$ fase e $19,1 \%$ a $8^{\text {a }}$. Quanto aos da medicina, todos cursavam a $11^{\text {a }}$ fase (Tabela 1 ).

Tabela 1 - Características sócio-demográficas dos acadêmicos de enfermagem e medicina. Florianópolis-SC, 2008

\begin{tabular}{lcc}
\hline \multirow{2}{*}{ Variáveis } & \multicolumn{2}{c}{ Acadêmicos } \\
\cline { 2 - 3 } & $\begin{array}{c}\text { Enfermagem } \\
(\mathrm{n}=47)\end{array}$ & $\begin{array}{c}\text { Medicina } \\
(\mathrm{n}=47)\end{array}$ \\
\hline Sexo & $\mathrm{f}(\%)$ & $\mathrm{f}(\%)$ \\
Feminino & $45(95,74)$ & $20(42,6)$ \\
Masculino & $1(2,13)$ & $27(57,4)$ \\
Não respondeu & $1(2,13)$ & - \\
Idade & & \\
$20-24$ & $35(74,5)$ & $28(59,6)$ \\
$25-29$ & $7(14,9)$ & $16(34,0)$ \\
$30-34$ & $2(4,25)$ & $3(6,4)$ \\
$35-40$ & $1(2,1)$ & - \\
Não respondeu & $2(4,25)$ & - \\
Estado marital & & \\
Solteiro & $39(83,0)$ & $45(95,7)$ \\
Casado & $3(6,4)$ & - \\
União consensual & $3(6,4)$ & $2(4,3)$ \\
Separado & $1(2,1)$ & - \\
Divorciado & $1(2,1)$ & - \\
Fase do curso & & - \\
5 $^{\text {a fase }}$ & $38(80,9)$ & - \\
8 fase & $9(19,1)$ & $47(100,0)$ \\
11ª fase & - &
\end{tabular}

O perfil desses estudantes de graduação é semelhante a estudos recentes com outros grupos de alunos. ${ }^{6-7} \mathrm{O}$ sexo feminino predomina sensivelmente na enfermagem, embora a população de acadêmicos de medicina também englobe um número bastante representativo de mulheres. A faixa de idade geral envolve adultos jovens, com menos de 24 anos, sendo que na medicina há um bom percentual de alunos com quase 30 anos, possivelmente justificado por estarem cursando períodos letivos mais avançados que os da enfermagem, pois enquanto os primeiros estavam concentradamente cursando a $5^{\text {a }}$ fase, os últimos cursavam a 11 a . Ressalta-se que estes dados eram esperados uma vez que a amostra foi intencional. O estado marital também concentra-se em torno dos que se declararam solteiros, embora entre os da enfermagem há maior concentração dos que responderam que são casados ou vivem em união consensual. Uma característica que parece estar aumentando nesta população. ${ }^{6}$

A maioria dos acadêmicos, tanto de enfermagem $(89,4 \%)$, como de medicina $(78,7 \%)$ referiu conhecer a filosofia da maternidade. Com relação ao acesso inicial à informação a respeito dessa filosofia, os acadêmicos de enfermagem referiram que o mesmo se deu, de forma mais frequente, nas aulas teóricas da disciplina da área obstétrica e neonatal (55,3\%), através dos professores (55,3\%) e por meio de murais da maternidade $(42,5 \%)$. Para os de medicina, o acesso ocorreu antes mesmo de iniciarem as atividades na maternidade $(40,4 \%)$ e nas aulas teóricas da disciplina da área obstétrica e neonatal (36,2\%) (Tabela 2).

Chama atenção a grande quantidade de acadêmicos de enfermagem e medicina que referiu conhecer a existência da filosofia da maternidade. Esta informação é relevante se considerarmos que as entradas dos mesmos nos cursos ocorrem com certa freqüência, ou seja, a cada semestre letivo, o que leva a uma grande demanda de novos estudantes a cada ano. Infere-se que se a grande maioria revelou que conhece a existência de uma filosofia de atenção a balizar a assistência, neste cenário hospitalar, é porque está sendo suprida de informações sobre o assunto durante o decorrer de seu processo formativo. Observou-se que a fonte de informações está mais frequentemente centrada nos cenários de aprendizagem teóricoprática, seja em salas de aula (previamente à entrada formal na maternidade), como é o caso da medicina, ou ainda, diretamente nos cenários assistenciais, como é o caso da enfermagem. Este detalhe relacionado à entrada no campo de atividades práticas pode estar relacionado ao projeto pedagógico de cada um desses cursos de graduação e não, necessariamente, à primazia 
que os alunos de medicina teriam sobre os da

desta informação, à exemplo de outros estudos enfermagem com relação à obtenção mais precoce

relacionados a cenários curriculares. ${ }^{6-8}$

Tabela 2 - Acesso dos acadêmicos de enfermagem e medicina à informação sobre a filosofia da maternidade. Florianópolis-SC, 2008

\begin{tabular}{lcc}
\hline \multirow{2}{*}{ Variáveis } & \multicolumn{2}{c}{ Acadêmicos } \\
\cline { 2 - 3 } & Enfermagem $(\mathrm{n}=47)$ & Medicina $(\mathrm{n}=47)$ \\
\hline Conhece a filosofia & & \\
Sim & $42(89,4)$ & $37(78,7)$ \\
Apenas ouvir falar & $4(8,5)$ & $6(12,8)$ \\
Não & - & $4(8,5)$ \\
Não respondeu & $1(2,1)$ & - \\
Acesso à informação sobre a filosofia & & \\
Nas aulas teóricas da disciplina & $26(55,3)$ & $17(36,2)$ \\
Através do(s) professor(es) da disciplina & $26(55,3)$ & $9(19,1)$ \\
Através de murais da maternidade & $20(42,5)$ & $14(29,8)$ \\
Antes de iniciar as atividades acadêmicas & $17(36,2)$ & $19(40,4)$ \\
Durante as atividades assistenciais & $16(34,0)$ & $7(14,9)$ \\
Nas aulas teórico-práticas desenvolvidas na maternidade & $16(34,0)$ & $10(21,3)$ \\
Em publicação sobre a maternidade & $5(10,6)$ & $1(2,1)$ \\
Através do coordenador da disciplina & $4(8,5)$ & $7(14,9)$ \\
Através dos profissionais da instituição & $1(2,1)$ & $11(23,4)$ \\
Através do preceptor & - & $12(25,5)$ \\
Outro & $3(6,4)$ & $1(2,1)$ \\
\hline Alguns acdo
\end{tabular}

* Alguns acadêmicos conheceram a filosofia por mais de uma fonte de informação, ${ }^{\dagger}$ Durante a realização do Trabalho de Conclusão de Curso e site do HU.

Os resultados apresentados nas Tabelas 3, 4 e 5 correspondem à avaliação dos acadêmicos de enfermagem e medicina acerca da operacionalização dos 12 princípios filosóficos nos setores da maternidade em que desenvolveram as atividades. Desta forma, em alguns setores há um elevado percentual de respostas na categoria Não Respondeu (N/R) ou Não se Aplica (N/A), o que significa que não estava previsto no plano de ensino da disciplina o desenvolvimento de atividades no(s) setor(es) ou não foi oportunizado.

De acordo com a Tabela 3, um elevado percentual (acima de $50 \%$ ) de acadêmicos de enfermagem considerou que o $1^{\circ}$ e o $3^{\circ}$ princípios são implementados plenamente no $\mathrm{CO}$, no $\mathrm{AC}$ e no CIAM; que o $10^{\circ}$ princípio é implementado plenamente no AC e no CIAM; e que o $12^{\circ}$ é implementado plenamente no $\mathrm{CO}$ e no $\mathrm{AC}$.

Quanto aos acadêmicos de medicina, os mesmos não responderam com tanta ênfase que tais princípios são assim operacionalizados. $\mathrm{Na}$ visão desses estudantes apenas o $1^{\circ}$ princípio foi considerado como sendo plenamente operacionalizado no AC $(65,1 \%)$, sendo que nos demais setores os percentuais não atingiram $50 \%$. Essa diferença de opinião entre os acadêmicos de enfermagem e medicina foi estatisticamente significativa $(\mathrm{CO}$, $p=0,026 ; A C, p=0,029$; CIAM, 0,003) (Tabela 3). Quanto à operacionalização do $3^{\circ}$ princípio, responderam que o mesmo é implementado na maioria dos setores (TO, CO, AC e AT). Chama a atenção que o $10^{\circ}$ princípio foi considerado plenamente operacionalizado apenas no $\mathrm{AC}(60,5 \%)$ e o $12^{\circ}$, apenas no CO $(60,5 \%)$ e no AC $(81,4 \%)$ (Tabela 3$)$.

Considerando que a pesquisa foi realizada em um HU, era esperado que o $1^{\circ}$ princípio, que preconiza a integração do ensino com a assistência, fosse plenamente implementado em todos os setores da maternidade. Esse fato foi confirmado pela maioria dos acadêmicos de enfermagem, no entanto, os de medicina consideraram que na maior parte dos setores não é colocado em prática ou apenas parcialmente. Esse resultado discrepante pode ser decorrente do próprio projeto pedagógico de cada curso. Os acadêmicos de enfermagem possuem um acompanhamento contínuo e direto de uma professora da disciplina durante o desenvolvimento das atividades assistenciais. Os de medicina estão, nessa fase, realizando o treinamento supervisionado em serviço, sendo orientados pelos médicos plantonistas e residentes. ${ }^{9}$ 
Tabela 3 - Opinião dos acadêmicos sobre a implementação dos princípios filosóficos relativos aos aspectos gerais da assistência. Florianópolis-SC, 2008

\begin{tabular}{|c|c|c|c|c|c|c|c|c|c|}
\hline \multirow{3}{*}{ Setores } & \multicolumn{9}{|c|}{ Acadêmicos } \\
\hline & \multicolumn{4}{|c|}{ Enfermagem $(n=47)$} & \multicolumn{5}{|c|}{ Medicina $(n=43)^{*}$} \\
\hline & $\begin{array}{l}\text { Sim } \\
f(\%)\end{array}$ & $\begin{array}{l}\text { Não } \\
f(\%)\end{array}$ & $\begin{array}{c}\text { Em parte } \\
f(\%)\end{array}$ & $\begin{array}{c}\mathrm{NR} \mathrm{A}^{\dagger} \\
\mathrm{f}(\%)\end{array}$ & $\begin{array}{l}\text { Sim } \\
f(\%)\end{array}$ & $\begin{array}{l}\text { Não } \\
f(\%)\end{array}$ & $\begin{array}{c}\text { Em parte } \\
f(\%)\end{array}$ & $\begin{array}{l}\text { NR/A } \\
f(\%)\end{array}$ & $\begin{array}{c}\mathrm{p} \\
\text { valor }^{+\dagger}\end{array}$ \\
\hline \multicolumn{10}{|c|}{$1^{\circ}$ princípio - Em se prestando assistência, se ensina } \\
\hline $\mathrm{TO}^{\ddagger}$ & $10(21,3)$ & - & $7(14,9)$ & $30(63,8)$ & $13(30,2)$ & $8(18,6)$ & $18(41,9)$ & $4(9,4)$ & 0,136 \\
\hline $\mathrm{CO}$ & $33(70,2)$ & $1(2,1)$ & $9(19,1)$ & $4(8,5)$ & $18(41,9)$ & $5(11,6)$ & $18(41,9)$ & $2(4,6)$ & 0,026 \\
\hline$A C \|$ & $40(85,1)$ & - & $6(12,8)$ & $1(2,1)$ & $28(65,1)$ & $2(4,7)$ & $13(30,2)$ & - & 0,029 \\
\hline ATा & $6(12,8)$ & $1(2,1)$ & $1(2,1)$ & $39(83,0)$ & $17(39,5)$ & $6(14,0)$ & $15(34,9)$ & $5(11,6)$ & 0,242 \\
\hline $\mathrm{CIAM}^{* *}$ & $30(63,8)$ & - & $3(6,4)$ & $14(29,8)$ & $11(25,6)$ & $3(7,0)$ & $7(16,3)$ & $22(51,2)$ & 0,003 \\
\hline
\end{tabular}

$3^{\circ}$ princípio - A atenção à saúde da mulher visa o ciclo gravídico-puerperal, considerando a gravidez como um processo e não como um evento

\begin{tabular}{lccccccccc}
\hline TO & $11(23,4)$ & $1(2,1)$ & $3(6,4)$ & $32(68,1)$ & $22(51,1)$ & $7(16,3)$ & $11(25,6)$ & $3(7,0)$ & 0,353 \\
CO & $30(63,8)$ & $1(2,1)$ & $12(25,5)$ & $4(8,5)$ & $30(69,8)$ & $4(9,3)$ & $7(16,3)$ & $2(4,6)$ & 0,917 \\
AC & $39(83,0)$ & - & $7(14,9)$ & $1(2,1)$ & $37(86,0)$ & $1(2,3)$ & $5(11,6)$ & - & 0,895 \\
AT & $5(10,6)$ & - & $3(6,4)$ & $39(83,0)$ & $25(58,1)$ & $4(9,3)$ & $8(18,6)$ & $6(14,0)$ & 0,890 \\
CIAM & $26(55,3)$ & - & $6(12,8)$ & $15(31,9)$ & $18(41,9)$ & $1(2,3)$ & $1(2,3)$ & $23(53,5)$ & 0,648 \\
\hline
\end{tabular}

$10^{\circ}$ princípio - A parturiente não deixará de ser assistida em face de problemas burocráticos, sendo que as rotinas deverão ser flexíveis

\begin{tabular}{lccccccccc}
\hline TO & $12(25,5)$ & $1(2,1)$ & $4(8,5)$ & $30(63,9)$ & $18(41,9)$ & $9(20,9)$ & $14(32,5)$ & $2(4,7)$ & 0,118 \\
CO & $23(48,9)$ & $1(2,1)$ & $17(36,2)$ & $6(12,8)$ & $19(44,2)$ & $9(20,9)$ & $13(30,2)$ & $2(4,7)$ & 0,507 \\
AC & $33(70,2)$ & $1(2,1)$ & $10(21,3)$ & $3(6,4)$ & $26(60,5)$ & $7(16,3)$ & $10(23,2)$ & - & 0,221 \\
AT & $6(12,8)$ & - & $3(6,4)$ & $38(80,8)$ & $16(37,2)$ & $9(20,9)$ & $12(27,9)$ & $6(14,0)$ & 0,373 \\
CIAM & $24(51,1)$ & - & $7(14,9)$ & $16(34,0)$ & $12(27,9)$ & $4(9,3)$ & $3(7,0)$ & $24(55,8)$ & 0,443 \\
\hline
\end{tabular}

$12^{\circ}$ princípio - A mulher deve permanecer internada o tempo suficiente para que sejam atendidas suas necessidades assistenciais e de educação em saúde

\begin{tabular}{lccccccccc}
\hline TO & $12(25,5)$ & - & $4(8,5)$ & $31(66,0)$ & $17(39,5)$ & $3(7,0)$ & $14(32,5)$ & $9(21,3)$ & 0,172 \\
CO & $30(63,8)$ & $1(2,1)$ & $11(23,4)$ & $5(10,6)$ & $26(60,5)$ & $2(4,7)$ & $11(25,6)$ & $4(9,4)$ & 0,823 \\
AC & $36(76,6)$ & $1(2,1)$ & $7(14,9)$ & $3(6,4)$ & $35(81,4)$ & $1(2,3)$ & $7(16,3)$ & - & 0,821 \\
AT & $5(10,6)$ & - & $1(2,1)$ & $41(87,2)$ & $16(37,2)$ & $3(7,0)$ & $9(20,9)$ & $15(34,9)$ & 0,462 \\
CIAM & $22(46,8)$ & $1(2,1)$ & $4(8,5)$ & $20(42,6)$ & $14(32,6)$ & $1(2,3)$ & $4(9,3)$ & $24(55,8)$ & 0,788 \\
\hline
\end{tabular}

* 4 acadêmicos de medicina não responderam a este quesito, justificando que não conheciam a filosofia da maternidade. ${ }^{\dagger}$ NR/A - Não Respondeu ou Não se Aplica, ₹ TO - Triagem Obstétrica, ${ }^{\S}$ CO - Centro Obstétrico, 11 AC - Alojamento Conjunto, ^ AT Ambulatório de Tocoginecologia, ${ }^{* *}$ CIAM - Central de Incentivo ao Aleitamento Materno. ${ }^{\dagger+}$ Teste qui-quadrado

Na ótica dos acadêmicos de enfermagem e medicina, ao se prestar assistência à mulher, nos diferentes setores da maternidade do HU/UFSC, considera-se a gravidez como um processo e não somente um evento ( $3^{\circ}$ princípio). Salienta-se que além de ser um aspecto assistencial muito positivo, também influencia na formação desses futuros profissionais que, se espera, sejam protagonistas de mudanças na assistência obstétrica. No paradigma da atenção humanizada, o nascimento é um processo no qual os profissionais são coadjuvantes e devem estar preparados para prestar assistência adequada, respeitando os significados que envolvem a experiência. ${ }^{10}$
No entanto, é preocupante que esses mesmos acadêmicos consideraram que o $10^{\circ}$ princípio, que recomenda a flexibilização das rotinas, tenha sido plenamente operacionalizado em apenas alguns setores (AC e CIAM), sendo que o CO não está incluído. No cotidiano do cuidado, as rotinas são, sem dúvida, fundamentais para a organização do serviço, mas, muitas vezes, os profissionais as colocam em prática de forma arbitrária, quando se sentem incapazes de lidar com determinadas situações que envolvem aspectos emocionais oriundos da experiência parturitiva. Este aspecto é bastante crítico, considerando-se os pressupostos de humanização da assistência em nível hospitalar. 
Resultados de uma pesquisa sobre humanização do parto apontou que os profissionais, diante da expressão de desespero da parturiente, se restringem a seguir rotinas, o que impede a participação da mulher e também do acompanhante, evidenciando a autoridade da equipe de saúde. ${ }^{11}$

Embora a implementação plena do $12^{\circ}$ princípio seja apenas no $\mathrm{AC}$ e $\mathrm{CO}$, isto não é preocupante, uma vez que esses são os setores em que a assistência requer um tempo de internação que seja adequado para o desenvolvimento das atividades assistenciais e de educação em saúde. Nos demais setores a mulher não permanece internada, pois se caracteriza como assistência ambulatorial (AT e TO) ou para atendimento de necessidades relacionadas ao aleitamento materno (CIAM).
Quanto à opinião sobre a implementação dos princípios filosóficos relacionados à atuação da equipe e à integração entre os profissionais e entre os setores (Tabela 4), um elevado percentual de acadêmicos de enfermagem (acima de 50\%) considerou que todos os princípios são praticados de forma plena no CO, AC e CIAM. Os de medicina responderam que o $6^{\circ}$ e o $9^{\circ}$ princípio são plenamente operacionalizados no $\mathrm{AC}, \mathrm{o} 7^{\circ}$ no $\mathrm{CO}$ e no $\mathrm{AC}$, e o $8^{\circ}$ no $\mathrm{CO}$, AC e CIAM. Chama a atenção o fato do $11^{\circ}$ princípio ter sido considerado implementado nos diversos setores, por menos de $50 \%$ desses acadêmicos e que, a operacionalização plena de todos os princípios na TO e no AT, também foram avaliados nessa mesma percentagem.

Tabela 4 - Opinião dos acadêmicos sobre a implementação dos princípios filosóficos relacionados à atuação da equipe e à integração entre os profissionais e entre os setores. Florianópolis-SC, 2008

Continua

\begin{tabular}{|c|c|c|c|c|c|c|c|c|c|}
\hline \multirow{3}{*}{ Setores } & \multicolumn{8}{|c|}{ Acadêmicos } & \\
\hline & \multicolumn{4}{|c|}{ Enfermagem $(n=47)$} & \multicolumn{5}{|c|}{ Medicina $(n=43)^{*}$} \\
\hline & $\begin{array}{l}\text { Sim } \\
f(\%)\end{array}$ & $\begin{array}{l}\text { Não } \\
f(\%)\end{array}$ & $\begin{array}{c}\text { Em parte } \\
f(\%)\end{array}$ & $\begin{array}{l}\mathrm{NR} \mathrm{A}^{\dagger} \\
\mathrm{f}(\%)\end{array}$ & $\begin{array}{l}\text { Sim } \\
f(\%)\end{array}$ & $\begin{array}{l}\text { Não } \\
f(\%)\end{array}$ & $\begin{array}{c}\text { Em parte } \\
f(\%)\end{array}$ & $\begin{array}{l}\text { NR/A } \\
f(\%)\end{array}$ & $\begin{array}{c}\mathrm{p} \\
\text { valor }^{\dagger+}\end{array}$ \\
\hline
\end{tabular}

$6^{\circ}$ princípio - A equipe interdisciplinar que presta assistência à mulher/recém-nascido/família deve atuar de forma integrada, visando um atendimento adequado

\begin{tabular}{lccccccccc}
\hline TO $^{\ddagger}$ & $12(25,5)$ & - & $3(6,4)$ & $32(68,1)$ & $16(37,2)$ & $11(25,6)$ & $13(30,2)$ & $3(7,0)$ & 0,019 \\
CO & $24(51,0)$ & $2(4,3)$ & $17(36,2)$ & $4(8,5)$ & $20(46,5)$ & $5(11,6)$ & $16(37,2)$ & $2(4,7)$ & 0,669 \\
AC $^{\prime \prime}$ & $32(68,1)$ & $1(2,1)$ & $13(27,7)$ & $1(2,1)$ & $34(79,1)$ & $4(9,3)$ & $5(11,6)$ & - & 0,434 \\
AT $^{\top}$ & $6(12,8)$ & - & $2(4,2)$ & $39(83,0)$ & $16(37,2)$ & $10(23,3)$ & $9(20,9)$ & $8(18,6)$ & 0,270 \\
CIAM $^{* *}$ & $24(51,1)$ & - & $8(17,0)$ & $15(31,9)$ & $17(39,5)$ & $3(7,0)$ & $3(7,0)$ & $20(46,5)$ & 0,823 \\
\hline
\end{tabular}

$7^{\circ}$ princípio - As atividades de ensino, pesquisa e extensão realizadas pela equipe interdisciplinar devem refletir atitudes de respeito ao ser humano e reverter em benefício de melhor assistência

\begin{tabular}{lccccccccc}
\hline TO & $11(23,4)$ & - & $5(10,6)$ & $31(66,0)$ & $21(48,8)$ & $6(14,0)$ & $13(30,2)$ & $3(7,0)$ & 0,417 \\
CO & $29(61,7)$ & $1(2,1)$ & $12(25,5)$ & $5(10,6)$ & $22(51,2)$ & $4(9,3)$ & $15(34,9)$ & $2(4,6)$ & 0,224 \\
AC & $33(70,2)$ & $1(2,1)$ & $10(21,3)$ & $3(6,4)$ & $29(67,4)$ & $2(4,7)$ & $12(27,9)$ & - & 0,587 \\
AT & $5(10,6)$ & - & $3(6,4)$ & $39(83,0)$ & $21(48,8)$ & $6(14,0)$ & $10(23,3)$ & $6(14,0)$ & 0,923 \\
CIAM & $26(55,3)$ & - & $5(10,6)$ & $16(34,0)$ & $14(32,6)$ & $2(4,6)$ & $2(4,6)$ & $25(58,2)$ & 0,882
\end{tabular}

$8^{\circ}$ princípio - A equipe deve exercer papel atuante na educação da mulher/acompanhante e grupo familiar, com vistas ao preparo e adaptação do aleitamento materno, desenvolvimento da confiança e capacidade de cuidar do filho, execução de cuidados básicos de saúde e planejamento familiar

\begin{tabular}{lccccccccc}
\hline TO & $5(10,6)$ & $2(4,3)$ & $9(19,1)$ & $31(66,0)$ & $15(34,9)$ & $10(23,2)$ & $14(32,6)$ & $4(9,3)$ & 0,844 \\
CO & $26(55,3)$ & $1(2,1)$ & $15(32,0)$ & $5(10,6)$ & $24(55,8)$ & $5(11,6)$ & $12(27,9)$ & $2(4,7)$ & 0,928 \\
AC & $44(93,6)$ & - & $2(4,3)$ & $1(2,1)$ & $34(79,1)$ & - & $9(20,9)$ & - & 0,040 \\
AT & $5(10,6)$ & - & $3(6,4)$ & $39(83,0)$ & $16(37,2)$ & $6(14,0)$ & $13(30,2)$ & $8(18,6)$ & 0,641 \\
CIAM & $32(68,1)$ & - & $1(2,1)$ & $14(29,8)$ & $22(51,2)$ & - & $1(2,3)$ & $20(46,5)$ & 0,638
\end{tabular}




\begin{tabular}{|c|c|c|c|c|c|c|c|c|c|}
\hline \multicolumn{10}{|c|}{$\begin{array}{l}9^{\circ} \text { princípio - Todo pessoal deve ter qualificação, treinamento e supervisão contínua e específica para pres- } \\
\text { tação da assistência a que tem direito a mãe, o recém-nascido e a família }\end{array}$} \\
\hline TO & $10(21,3)$ & -- & $6(12,8)$ & $31(65,9)$ & $13(30,2)$ & $7(16,3)$ & $21(48,8)$ & $2(4,7)$ & 0,067 \\
\hline $\mathrm{CO}$ & $32(68,1)$ & $2(4,3)$ & $9(19,1)$ & $4(8,5)$ & $17(39,5)$ & $2(4,7)$ & $22(51,1)$ & $2(4,7)$ & 0,004 \\
\hline$A C$ & $35(74,5)$ & $2(4,3)$ & $9(19,1)$ & $1(2,1)$ & $24(55,8)$ & $4(9,3)$ & $14(32,6)$ & $1(2,3)$ & 0,096 \\
\hline AT & $7(14,9)$ & $1(2,1)$ & $1(2,1)$ & $38(80,9)$ & $21(48,8)$ & $3(7,0)$ & $13(30,2)$ & $6(14,0)$ & 0,436 \\
\hline CIAM & $29(61,7)$ & - & $4(8,5)$ & $14(29,8)$ & $15(34,9)$ & $3(7,0)$ & $3(7,0)$ & $22(51,1)$ & 0,246 \\
\hline \multicolumn{10}{|c|}{$\begin{array}{l}11^{\circ} \text { princípio - } 0 \text { desenvolvimento de atividades será de forma integrada quanto às unidades que operam na } \\
\text { maternidade, ou com ela se relacionem }\end{array}$} \\
\hline TO & $12(25,5)$ & - & $5(10,6)$ & $30(63,9)$ & $15(34,9)$ & $6(13,9)$ & $19(44,2)$ & $3(7,0)$ & 0,067 \\
\hline $\mathrm{CO}$ & $29(61,7)$ & $1(2,1)$ & $13(27,7)$ & $4(8,5)$ & $18(41,9)$ & $5(11,6)$ & $17(39,5)$ & $3(7,0)$ & 0,004 \\
\hline$A C$ & $34(72,3)$ & $1(2,1)$ & $11(23,4)$ & $1(2,1)$ & $21(48,8)$ & $3(7,0)$ & $18(41,9)$ & $1(2,3)$ & 0,096 \\
\hline AT & $6(12,8)$ & - & $2(4,2)$ & $39(83,0)$ & $18(41,9)$ & $5(11,6)$ & $16(37,2)$ & $4(9,3)$ & 0,436 \\
\hline CIAM & $26(55,3)$ & - & $7(14,9)$ & $14(29,8)$ & $9(20,9)$ & $4(9,3)$ & $9(20,9)$ & $21(48,9)$ & 0,246 \\
\hline
\end{tabular}

* 4 acadêmicos de medicina não responderam a este quesito, justificando que não conheciam a filosofia da maternidade. ${ }^{\dagger} \mathrm{NR} / \mathrm{A}-\mathrm{Não}$ Respondeu ou Não se Aplica, ₹ TO - Triagem Obstétrica, ${ }^{\S}$ CO - Centro Obstétrico, " AC - Alojamento Conjunto, " AT - Ambulatório de Tocoginecologia, ${ }^{* *}$ CIAM - Central de Incentivo ao Aleitamento Materno. ${ }^{\text {tt }}$ Teste qui-quadrado

Como já pontuado anteriormente, as experiências do acadêmico que desenvolve atividades na maternidade são influenciadas pela forma como as práticas de ensino são organizadas e conduzidas nos diferentes setores, assim como pelo exemplo prático dos profissionais de cada uma das categorias, pois isso se tornará uma referência (modelo) para a sua atuação futura como profissional. Esses aspectos podem ter contribuído para que os acadêmicos de enfermagem tenham tido uma opinião mais positiva sobre a implementação de todos esses princípios nos setores em que tiveram oportunidade de atuar (CO, AC e CIAM). No entanto, acredita-se que isso não tenha mascarado ou enviesado a real percepção desses acadêmicos. Faz-se essa consideração pelo fato de que, em outra pesquisa, os acadêmicos de enfermagem expressaram descontentamento com o cuidado prestado durante o processo de parturição, sendo capazes de perceber que a prática não era condizente com o preconizado na disciplina e pelas recomendações do MS. ${ }^{5}$

A avaliação dos acadêmicos de medicina não foi tão positiva como os de enfermagem, havendo diferença estatisticamente significativa quanto à operacionalização do $6^{\circ}$ princípio na $\mathrm{TO}(\mathrm{p}=0,019)$, $8^{\circ}$ princípio no $\mathrm{AC}(\mathrm{p}=0,040), 9^{\circ}$ princípio no $\mathrm{CO}$ $(\mathrm{p}=0,004)$ e $11^{\circ}$ princípio no $\mathrm{CO}(\mathrm{p}=0,004)$ (Tabela 4). O princípio relacionado ao desenvolvimento de atividades de forma integrada entre os serviços que compõem a maternidade ( $11^{\circ}$ princípio), foi considerado pouco implementado. Isso mostra uma fragilidade nas relações que se estabelecem entre os setores, que foi percebida por esses aca- dêmicos. A não operacionalização desse princípio pode estar interferindo negativamente no processo de formação profissional desses alunos e também na concretização de outros princípios, em especial o que preconiza que a equipe interdisciplinar deve atuar de forma integrada, visando um atendimento adequado ( $6^{\circ}$ princípio), o qual foi considerado plenamente praticado apenas no AC. O atendimento integral ao cliente requer que a equipe atue de forma articulada e, para tanto, é necessário investir na comunicação entre os profissionais, valorizar iniciativas e compartilhar idéias. ${ }^{12}$

Merece ressaltar que a TO e o AT foram os setores em que todos os princípios filosóficos relacionados à atuação da equipe e à integração entre os profissionais foram considerados implementados parcialmente ou não implementados. Esse resultado é temível, uma vez que são setores em que o atendimento prestado não se encerra per se, já que as usuárias, em algum momento, necessitarão de encaminhamento para os demais setores da maternidade.

Quanto à opinião dos acadêmicos sobre a implementação dos princípios filosóficos relacionados aos direitos dos usuários $\left(2^{\circ}, 4^{\circ}\right.$ e $\left.5^{\circ}\right)$ (Tabela 5) a maioria dos acadêmicos de enfermagem (mais de 50\%) respondeu que todos são operacionalizados plenamente no $\mathrm{CO}$ e no $\mathrm{AC}$, sendo que o $2^{\circ}$ é praticado plenamente também no CIAM. Quanto aos da medicina, a maioria (mais de $50 \%$ ) respondeu que o $2^{\circ}$ e o $4^{\circ}$ princípios são plenamente concretizados no $\mathrm{CO}$ e no $\mathrm{AC}$, e o 5', apenas no AC. Entretanto, os acadêmicos 
de enfermagem consideraram que o $4^{\circ}$ princípio é mais operacionalizado no $\mathrm{CO}$ e $\mathrm{AC}$ do que os de medicina $(C O, p=0,029 ; A C, p=0,004)$. Cabe destacar que menos de $50 \%$ dos acadêmicos de medicina considerou que todos os princípios são operacionalizados na TO e no AT.

Tabela 5 - Opinião dos acadêmicos sobre a implementação dos princípios filosóficos relacionados aos direitos dos usuários. Florianópolis-SC, 2008

\begin{tabular}{|c|c|c|c|c|c|c|c|c|c|}
\hline \multirow[t]{4}{*}{ Setores } & \multicolumn{9}{|c|}{ Acadêmicos } \\
\hline & \multicolumn{4}{|c|}{ Enfermagem $(n=47)$} & \multicolumn{5}{|c|}{ Medicina $(n=43)^{*}$} \\
\hline & Sim & Não & Em parte & $\mathrm{NR} / \mathrm{A}^{\dagger}$ & Sim & Não & Em parte & NR/A & \\
\hline & $f(\%)$ & $f(\%)$ & $f(\%)$ & $f(\%)$ & $f(\%)$ & $f(\%)$ & $f(\%)$ & $f(\%)$ & \\
\hline \multicolumn{10}{|c|}{$\begin{array}{l}2^{\circ} \text { princípio - É direito de toda mulher/recém-nascido (RN)/família, no processo de gravidez, parto e puerpé- } \\
\text { rio, receber atendimento personalizado que garanta uma assistência adequada, nos aspectos biológicos, } \\
\text { sociais, psicológicos e espirituais }\end{array}$} \\
\hline $\mathrm{TO}^{\ddagger}$ & $13(27,6)$ & - & $2(4,3)$ & $32(68,1)$ & $17(39,5)$ & $3(7,0)$ & $21(48,8)$ & $2(4,7)$ & 0,006 \\
\hline $\mathrm{CO}^{\S}$ & $28(59,6)$ & - & $15(31,9)$ & $4(8,5)$ & $23(53,5)$ & $2(4,7)$ & $16(37,2)$ & $2(4,7)$ & 0,533 \\
\hline$A C^{\prime \prime}$ & $41(87,2)$ & - & $5(10,6)$ & $1(2,1)$ & $32(74,4)$ & $1(2,3)$ & $10(23,3)$ & - & 0,126 \\
\hline AT & $6(12,8)$ & - & $2(4,3)$ & $39(83,0)$ & $18(41,9)$ & $1(2,3)$ & $19(44,2)$ & $5(11,6)$ & 0,247 \\
\hline $\mathrm{CIAM}^{* *}$ & $28(59,6)$ & - & $5(10,6)$ & $14(29,8)$ & $18(41,9)$ & $1(2,3)$ & $3(7,0)$ & $21(48,8)$ & 0,940 \\
\hline \multicolumn{10}{|c|}{$\begin{array}{l}4^{\circ} \text { princípio - Na atenção à saúde da mãe, RN e família, na gravidez, parto e puerpério, se considera a im- } \\
\text { portância do papel do pai, sua presença e participação }\end{array}$} \\
\hline TO & $6(12,8)$ & $1(2,1)$ & $8(17,0)$ & $32(68,1)$ & $21(48,8)$ & $4(9,3)$ & $14(32,6)$ & $4(9,3)$ & 0,543 \\
\hline $\mathrm{CO}$ & $39(83,0)$ & - & $4(8,5)$ & $4(8,5)$ & $27(62,8)$ & $1(2,3)$ & $11(25,6)$ & $4(9,3)$ & 0,029 \\
\hline$A C$ & $43(91,5)$ & - & $3(6,4)$ & $1(2,1)$ & $27(62,8)$ & $1(2,3)$ & $12(27,9)$ & $3(7,0)$ & 0,004 \\
\hline AT & $5(10,6)$ & -- & $2(4,3)$ & $40(85,1)$ & $19(44,2)$ & $4(9,3)$ & $12(27,9)$ & $8(18,6)$ & 0,675 \\
\hline CIAM & $22(46,8)$ & -- & $10(21,3)$ & $15(31,9)$ & $13(30,2)$ & $1(2,3)$ & $6(14,0)$ & $23(53,5)$ & 0,981 \\
\hline
\end{tabular}

$5^{\circ}$ princípio - $\mathrm{O}$ sistema de alojamento conjunto facilita a criação e aprofundamento de laços mãe-RNfamília, favorecendo a vinculação afetiva, a compreensão do processo de crescimento e desenvolvimento, a participação ativa e a educação para a saúde dos elementos mencionados

\begin{tabular}{llrlllllll}
\hline TO & $8(17,0)$ & $1(2,1)$ & $4(8,5)$ & $34(72,3)$ & $17(39,5)$ & $5(11,6)$ & $10(23,3)$ & $11(25,6)$ & 0,854 \\
CO & $28(59,6)$ & -- & $6(12,8)$ & $13(27,6)$ & $20(46,5)$ & $2(4,7)$ & $10(23,2)$ & $11(25,6)$ & 0,125 \\
AC & $43(91,5)$ & -- & $2(4,3)$ & $2(4,3)$ & $38(88,4)$ & -- & $5(11,6)$ & -- & 0,394 \\
AT & $6(12,8)$ & -- & $1(2,1)$ & $40(85,1)$ & $19(44,2)$ & $4(9,3)$ & $6(14,0)$ & $14(32,6)$ & 0,559 \\
CIAM & $23(48,9)$ & -- & $2(4,3)$ & $22(46,8)$ & $16(37,2)$ & -- & $2(4,6)$ & $25(58,1)$ & 0,852
\end{tabular}

* 4 acadêmicos de medicina não responderam a este quesito, justificando que não conheciam a filosofia da maternidade. ${ }^{\dagger} \mathrm{NR} / \mathrm{A}-\mathrm{Não}$ Respondeu ou Não se Aplica, ‡ TO - Triagem Obstétrica, ${ }^{\S}$ CO - Centro Obstétrico, "I AC - Alojamento Conjunto, ^ AT - Ambulatório de Tocoginecologia, ${ }^{* \star}$ CIAM - Central de Incentivo ao Aleitamento Materno. ${ }^{\text {tt }}$ Teste qui-quadrado

De modo geral a avaliação dos acadêmicos de enfermagem e medicina sobre todos os princípios foi positiva para os setores de mencionados anteriormente, mas cabe destacar que o princípio relacionado ao direito dos usuários em receber um atendimento personalizado ( $2^{\circ}$ princípio) foi considerado como plenamente implementado no AC por um elevado percentual de acadêmicos (87,2\% de enfermagem, $74,4 \%$ de medicina), assim como o $5^{\circ}$ princípio, que trata da importância do sistema de AC na vinculação afetiva mãe-filho e na educação para a saúde $(91,5 \%$ de enfermagem, $88,4 \%$ de medicina). Esses dados permitem infe- rir que o empenho dos profissionais em praticar esse princípio pode ter gerado essa percepção tão positiva. Espera-se que a clientela assistida no AC esteja beneficiando-se com isso, uma vez que outros estudos ${ }^{13-15}$ têm apontado que as puérperas não recebem orientações suficientes durante a internação no AC, gerando uma desinstrumentalização para cuidar de si e do recém-nascido.

Entretanto, para os acadêmicos de medicina, a implementação desses princípios nos setores de AT e TO é deficitária, com especial destaque para o $2^{\circ}$ princípio, que foi considerado o menos praticado. Esta constatação é, de certa forma, preocu- 
pante, uma vez que o Projeto Político-Pedagógico do Curso de Graduação está pautado em diretrizes que visam promover o estudante de forma significativa e próxima dos desafios que enfrentará na realidade profissional, onde atuará depois de concluída a graduação. ${ }^{9}$ Este entendimento, certamente, implica em habilitar o futuro profissional para o desenvolvimento de capacidades não apenas técnicas, mas também afetivas, políticas e relacionais. Se o acadêmico, como ser aprendente de seu futuro cabedal profissional, não tiver a oportunidade de praticar ou ver operacionalizada a assistência individualizada, integral e centrada nos direitos da clientela, como poderá desempenhar este papel mais tarde, no cotidiano profissional? Este é um exemplo de algumas preocupações provocadas pelos resultados desta pesquisa e que precisarão ser investigadas, futuramente, com maior profundidade e especificidade.

A implementação do $4^{\circ}$ princípio, que preconiza a importância da presença e da participação do pai do bebê (ou acompanhante) durante a atenção à mulher na gravidez, parto e puerpério, foi destacada pelos acadêmicos com elevados percentuais no AC (91,5\% de enfermagem, $62,8 \%$ de medicina) e no CO (83,0\% de enfermagem, $62,8 \%$ de medicina. Esse resultado é extremamente relevante, uma vez que tal princípio inspirou a elaboração ${ }^{16}$ da Lei No 12.333 , de 12 de março de 2002, que assegurou o acompanhante no parto em todos os hospitais públicos e conveniados no estado de Santa Catarina e que contribui para a Lei ${ }^{\circ}$ 11.108, de 2005, ${ }^{16-17}$ que estende esse direito a todo o país, conhecida como "Lei do acompanhante". ${ }^{17}$ Isso mostra que o princípio continua merecendo destaque, não apenas por seu pioneirismo e repercussão, mas também pela sua manutenção. De certa maneira isso também se caracteriza como uma limitação do presente estudo. Este aspecto, inclusive, diz respeito a uma limitação mais global desta pesquisa, que permite observar que as taxas de respostas obtidas $(61,8 \%$ entre os graduandos em enfermagem potencialmente elegíveis e $50 \%$ entre os acadêmicos de medicina) limitam a extrapolação dos resultados para o universo de estudantes dos cursos.

\section{CONCLUSÕES}

Em termos globais, os acadêmicos identificam que os princípios filosóficos relativos aos aspectos gerais da assistência são implementados de modo mais concreto no CO, no AC e no CIAM, ou seja, em setores considerados com maior fluxo de mu- lheres, recém-nascidos e acompanhantes usuários da maternidade. Isso influencia positivamente na assistência, uma vez que se trata de princípios cujos teores se relacionam com a organização e planejamento da assistência. Destaca-se, contudo, nesta apreciação, o princípio que diz respeito à flexibilidade das rotinas ( $10^{\circ}$ princípio), pois os acadêmicos avaliam que o mesmo não é operacionalizado como deveria no $\mathrm{CO}$, no AT e no TO. Esse resultado merece ser melhor discutido com as equipes da maternidade, visando identificar estratégias que auxiliem no enfrentamento do problema.

A implementação do segundo conjunto de princípios, voltados à atuação da equipe e à integração dos profissionais entre os setores, é avaliada pela maioria dos acadêmicos de modo positivo. Contudo, ressalta-se que o $11^{\circ}$ princípio, relacionado especificamente com a integração entre os setores da maternidade, assim como o $6^{\circ}$, que focaliza a integração entre os membros da equipe, apresentam alguns problemas de operacionalização nos diversos setores, especialmente no TO e no $\mathrm{AT}$, o que também requer uma discussão e reflexão ampla com os profissionais envolvidos.

O terceiro conjunto de princípios, voltado aos direitos dos usuários, é considerado por grande parte dos acadêmicos, como sendo concretizado nos diversos setores, com destaque para o AC e o $\mathrm{CO}$, no tangente ao princípio que preconiza a importância da participação do pai do bebê e/ou acompanhante. Ainda, neste conjunto, os acadêmicos de medicina identificaram fragilidade de implementação, tanto na TO, quanto no AT.

Os aspectos negativos com relação à operacionalização da filosofia da maternidade na TOe no AT foram salientados pelos acadêmicos de medicina, mais do que os de enfermagem, uma vez que os mesmos desenvolvem atividades teórico-práticas em tais setores, de forma mais sistemática e frequente. Em contrapartida, a avaliação da filosofia no CIAM deu-se quase que exclusivamente sob a ótica dos acadêmicos de enfermagem, pelos mesmos motivos. De certa maneira isso também se caracteriza como uma limitação do presente estudo.

Esses resultados deixam entrever que, apesar dos acadêmicos perceberem avanços na atenção humanizada às famílias que experienciam o processo do nascimento no âmbito institucional, também são indicadores de que a operacionalização de uma filosofia plena de atenção ao parto e nascimento, ainda permanece um desafio a ser superado.

Considerando-se a inexistência de estudos brasileiros publicados envolvendo percepções de 
acadêmicos acerca de avaliações da assistência em saúde, assim como avaliações de instituições de saúde, os resultados desta pesquisa possuem valor redobrado. No contexto pesquisado, os acadêmicos de enfermagem e medicina avaliam que a operacionalização da filosofia possui diversas interpretações, ora colocando em foco o conjunto dos princípios filosóficos, ora analisando a implementação dos mesmos, em cada um dos setores que integram a maternidade do HU/UFSC.

\section{REFERÊNCIAS}

1. Tanaka OU, Melo C. Uma proposta de abordagem transdisciplinar para avaliação em saúde. Interface - Comunic Saúde Educ. 2000 Ago; 4(7):113-8.

2. World Health Organization, Pan American Health Organization. Joint interregional conference on appropriate technology for birth. Copenhagen (DK): World Health Organization Regional Office for Europe; 1985.

3. Brüggemann OM, Knobel R, Siebert ERC, Boing AF, Andrezzo HFA. Parto vertical na maternidade do hospital universitário da UFSC: série histórica, 1996 a 2005. Rev Bras Saúde Matern Infant. 2009 Abr-Jun; 9(2):189-96.

4. Siebert ERC. Incorporação da filosofia de atendimento da maternidade de um hospital universitário pelos profissionais do centro obstétrico [dissertação]. Florianópolis (SC): Universidade Federal de Santa Catarina, Programa de Pós-Graduação em Enfermagem; 2004.

5. Bicchieri T, Silva LR. A percepção dos acadêmicos de enfermagem sobre o cuidado à mulher no trabalho de parto e nascimento: uma abordagem qualitativa. Esc. Anna Nery R Enferm. 2006 Ago; 10(2):258-65.

6. Furegato ARF, Santos JLF, Silva EC. Depressão entre estudantes de enfermagem relacionada a auto-estima, a percepção da sua saúde e interesse por saúde mental. Rev Latino-am Enfermagem. 2008 Mar-Abr; 16(2):198-204.

7. Mesquita EM, Nunes AJ, Cohen C. Avaliação das atitudes dos estudantes de medicina frente ao abuso de drogas por colegas do meio acadêmico. Rev Psiq Clin. 2008; 35(supl 1):8-12.
8. Bicchieri T, Silva LR. A percepção dos acadêmicos de enfermagem sobre o cuidado à mulher no trabalho de parto e nascimento: uma abordagem qualitativa. Esc Anna Nery Rev Enferm. 2006 Ago; 10(2):258-65.

9. Curso de Medicina - Universidade Federal de Santa Catarina [página na Internet]. Florianópolis: UFSC; 2009 [acesso 2009 Jul 1]. Disponível em: http:// www.medicina.ufsc.br/sobre/historico.php

10. Wolff LR, Moura MAV. A institucionalização do parto e a humanização da assistência: revisão de literatura. Esc Anna Nery Rev Enferm. 2004 Ago; 8(2):279-85.

11. Reis AE, Patrício ZM. Aplicação das ações preconizadas pelo Ministério da Saúde para o parto humanizado em um hospital de Santa Catarina. Ciênc Saúde Coletiva. 2005 Set-Dez; 10(supl1):221-30.

12. Backes DS, Lunardi Filho WD, Lunardi V. O processo de humanização do ambiente hospitalar centrado no trabalhador. Rev Esc Enferm. USP. 2004 Jun; 40(2):221-7.

13. Bulhosa MS, Santos MG, Lunardi VL. Percepção de puérperas sobre o cuidado de enfermagem em unidade de alojamento conjunto. Cogitare Enferm. 2005 Jan-Abr; 10(1):42-7.

14. Parada CMGL, Tonete VLP. O cuidado em saúde no ciclo gravídico-puerperal sob a perspectiva de usuárias de serviços públicos. Interface - Comunic Saúde Educ. 2008 Jan-Mar; 12(24):35-46.

15. Monticelli M, Elsen I. A cultura como obstáculo: percepções da enfermagem no cuidado às famílias em alojamento conjunto. Texto Contexto Enferm. 2006 Jan-Mar; 15(1):115-21.

16. Brüggemann OM, Parpinelli MA, Osis MJD. Evidências sobre o suporte durante o trabalho de parto/parto: uma revisão da literatura. Cad Saúde Pública. 2005 Set-Out; 21(5):1316-27.

17. Brasil. Lei No 11.108: altera a Lei No 8.080, de 19 de setembro de 1990, para garantir às parturientes $\mathrm{o}$ direito à presença de acompanhante durante $\mathrm{o}$ trabalho de parto, parto e pós-parto imediato, no âmbito do Sistema Único de Saúde - SUS. Diário Oficial da União, Brasília, 2005. 